Cite this: RSC Adv., 2014, 4, 10856

Received 28th November 2013 Accepted 24th January 2014

DOI: $10.1039 / c 3 r a 47128 d$

www.rsc.org/advances

\section{Facile preparation of indoxyl- and nitrophenyl glycosides of lactosamine and isolactosamine $\uparrow$}

\author{
Stephan Böttcher and Joachim Thiem* \\ The synthesis of the novel indoxyl glycosides of $N$-acetyl-lactosamine (X-LacNAc) and N-acetyl- \\ isolactosamine (X-LNB) is reported employing glycosyl chlorides in a facile phase transfer glycosylation, \\ followed by mild decarboxylation and finally deacetylation. Correspondingly the ortho-nitrophenol and \\ para-nitrophenol glycosides of LacNAC and LNB could be obtained.
}

\section{Introduction}

$N$-Acetyl-lactosamine (LacNAc, Gal $\beta 1-4 \mathrm{GlcNAc}$ ) and $N$-acetylisolactosamine (LNB, Galß1-3GlcNAc) are integral parts of biologically very important glycostructures. Both represent essential subunits of complex milk oligosaccharides ${ }^{\mathbf{1 , 2}}$ as well as of antigens ${ }^{3-5}$ and glycoconjugates. ${ }^{6,7}$

Access to samples or intermediates of complex glycostructures is still rather difficult. The chemical synthesis of complex saccharide structures is loaded with barriers, regarding protecting group chemistry as well as control of stereo- and regio-chemistry. Enzymatic syntheses need fewer steps, are often highly stereo- and regio-selective and are therefore superior if available. Thus, these approaches complement the array of methods, however, often enzymes are not available or difficult to isolate, purify or handle.

A convenient method for screening of glycosidases as well as transglycosidases is to use indoxyl glycosides (Fig. 1). Indoxyl is released by enzymatic cleavage of the glycosidic linkage and then rapidly oxidized, e.g. by atmospheric oxygen, to an indigo type dye. Common substrates are halogenated, as the substitution pattern determines colour and physical properties of the resulting indigo dye. The most common pattern of indoxyls are 5-bromo-4-chloro, 5-bromo and 5-bromo-6-chloro derivatives. ${ }^{8}$ Nitrophenol glycosides in turn are widely utilised compounds for activity measurements and are employed as convenient donor substrates in enzymatic syntheses. ${ }^{\mathbf{9 - 1 2}}$

Here we wish to report convenient syntheses of novel indoxyl glycosides of $N$-acetyl-lactosamine (X-LacNAc) and $N$-acetyl-isolactosamine (X-LNB), as well as the synthesis of para-nitrophenyl and ortho-nitrophenyl glycosides of both disaccharides.

The peracetates of LacNAc and LNB were converted into the corresponding glycosyl chlorides. The crude products could be

Department of Chemistry, Faculty of Science, University of Hamburg, Martin-Luther-King-Platz 6, 20146 Hamburg, Germany. E-mail: thiem@chemie. uni-hamburg.de

$\dagger$ Electronic supplementary information (ESI) available: NMR data for all new compounds. See DOI: 10.1039/c3ra47128d

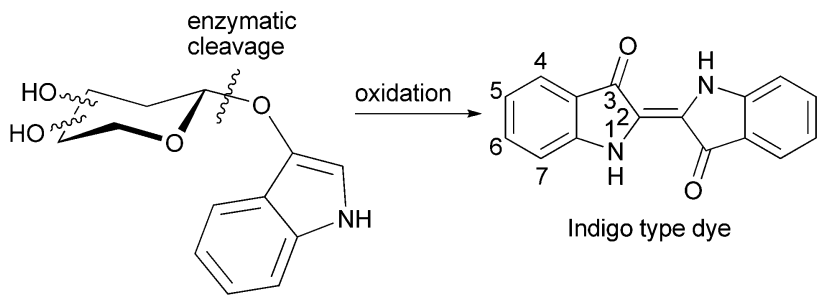

Fig. 1 Monitoring of glycosidase activity employing indoxyl glycosides.

used after washing directly without further workup for phase transfer glycosylation with the respective nitrophenol acceptor. The $o$ NP and $p$ NP glycosides were obtained after Zemplén deacetylation ${ }^{13}$ in good overall yields. Preparation of the indoxyl glycosides followed our recently developed approach. In a phase transfer glycosylation indoxylic acid allyl esters were used as acceptors, followed by selective mild silver mediated decarboxylation and finally Zemplén deacetylation. ${ }^{\mathbf{1 4 , 1 5}}$

\section{Results and discussion}

The synthetic route for the preparation of LNB- $o \mathrm{NP} / p \mathrm{NP}(\mathbf{5 a} / \mathbf{b})$ and LacNAc- $o \mathrm{NP} / p \mathrm{NP}(\mathbf{5} \mathbf{c} / \mathbf{d})$ is summarized in Scheme 1.

The peracetates $\mathbf{2 a} / \mathbf{b}$ were treated with acetyl chloride and methanol under argon atmosphere to give the glycosyl chlorides 3a/b. These donors were dried and washed to remove acetyl chloride, and were then subjected to phase transfer glycosylation without further workup to yield peracetylated LNB- $p$ NP (4a, $40 \%$ ), LNB- $o$ NP (4b, 34\%), LacNAc- $p$ NP (4c, 45\%), LNB- $o$ NP (4d, $44 \%$ ) via two steps. Finally, the acetyl protecting groups were removed by Zemplén deacetylation. ${ }^{\mathbf{1 3}}$ In case of $\mathbf{5 d}$ the reaction mixture needed to be kept at $45{ }^{\circ} \mathrm{C}$ overnight to achieve complete deacetylation. All glycosides crystallised during deacetylation and were washed after filtration with a small amount of cold methanol to give very pure $\mathbf{5 a - d}$ in good yields. 


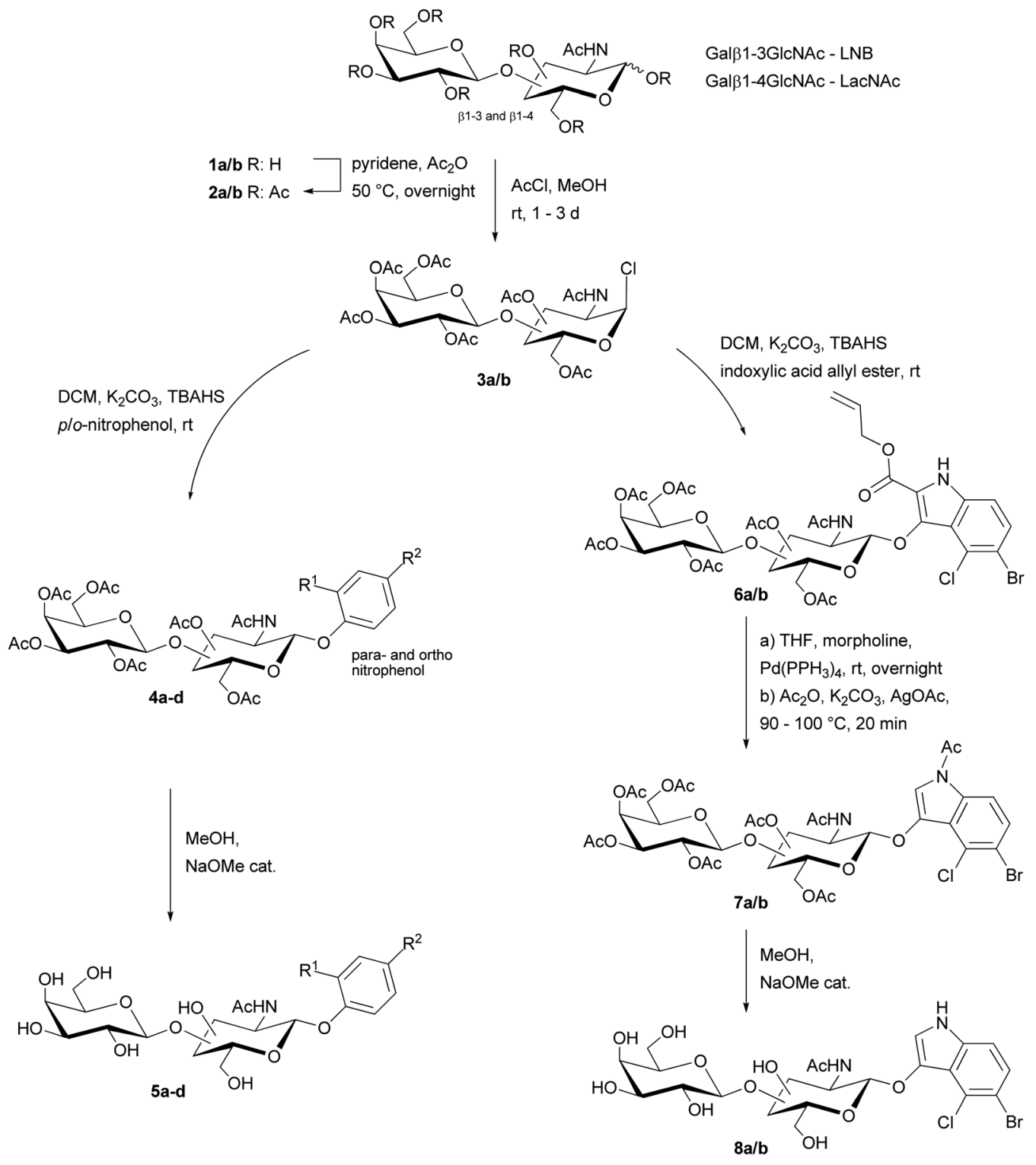

\begin{tabular}{|c|c|c|c|c|c|c|c|c|c|c|c|c|c|c|c|c|}
\hline & $2 a$ & $2 b$ & $4 a$ & $4 b$ & $4 c$ & $4 d$ & $5 \mathbf{a}$ & $5 b$ & $5 c$ & $5 d$ & $\mathbf{6 a}$ & $6 b$ & $7 \mathbf{a}$ & $7 b$ & $8 a$ & $8 b$ \\
\hline Glycoside & $\beta 1-3$ & $\beta 1-4$ & $\beta 1-3$ & $\beta 1-3$ & $\beta 1-4$ & $\beta 1-4$ & $\beta 1-3$ & $\beta 1-3$ & $\beta 1-4$ & $\beta 1-4$ & $\beta 1-3$ & $\beta 1-4$ & $\beta 1-3$ & $\beta 1-4$ & $\beta 1-3$ & $\beta 1-4$ \\
\hline Yield [\%] & 95 & 97 & $40 *$ & $34 *$ & $45^{*}$ & $44^{*}$ & 87 & 80 & 71 & 80 & $46^{*}$ & $45^{*}$ & 85 & 62 & 75 & 73 \\
\hline $\mathrm{R}^{1}$ & - & - & $\mathrm{H}$ & $\mathrm{NO}_{2}$ & $\mathrm{H}$ & $\mathrm{NO}_{2}$ & $\mathrm{H}$ & $\mathrm{NO}_{2}$ & $\mathrm{H}$ & $\mathrm{NO}_{2}$ & - & - & - & - & - & - \\
\hline $\mathrm{R}^{2}$ & - & - & $\mathrm{NO}_{2}$ & $\mathrm{H}$ & $\mathrm{NO}_{2}$ & $\mathrm{H}$ & $\mathrm{NO}_{2}$ & $\mathrm{H}$ & $\mathrm{NO}_{2}$ & $\mathrm{H}$ & - & - & - & - & - & - \\
\hline
\end{tabular}

Scheme 1 Synthesis of p/o-nitrophenol glycosides of isolactosamine ( $5 a / b)$ and lactosamine $(5 c / d)$ as well as indoxyl glycosides of isolactosamine (8a) and lactosamine (8b). *Yield via two steps.

The synthesis of the indoxyl glycosides $\mathbf{8 a} / \mathbf{b}$ was again based on the conversion of the respective peracetate $2 \mathbf{a}$ or $2 \mathbf{b}$ to the crude glycosyl chlorides $\mathbf{3 a} / \mathbf{b}$. Phase transfer glycosylation under common phase transfer conditions gave $\mathbf{6 a}(46 \%)$ and $\mathbf{6 b}$ (45\%). Then selective allyl ester deprotection with $\mathrm{Pd}\left(\mathrm{PPh}_{3}\right)_{4}$ and morpholine in $\mathrm{THF}^{16}$ followed by mild silver mediated decarboxylation $^{14,15}$ gave the acetylated glycosides 7 a (85\%) ad $\mathbf{7 b}(62 \%)$. Finally Zemplén deacetylation ${ }^{13}$ was used to give the novel indoxyl glycosides X-LNB in $75 \%$ and X-LacNAc in $73 \%$ yield. 


\section{Conclusions}

Within this work we could elaborate facile syntheses of novel indoxyl glycosides X-LAcNAc and X-LNB as well as the corresponding ortho- and para-nitrophenyl glycosides of LacNAc and LNB. Both reaction pathways could be carried out in good yields, employing facile phase transfer glycosylation of the crude glycosyl chloride donors, straight forward workup and crystallization.

\section{Experimental section}

\section{General remarks}

All reagents were purchased from commercial sources and used as received. TLC was performed on Merck silica gel $60 \mathrm{~F}_{254}$ plates. Compounds were detected by UV and/or by treatment with $\mathrm{EtOH} / \mathrm{H}_{2} \mathrm{SO}_{4}(9: 1)$ and subsequent heating. Column chromatography was performed with Merck/Fluka silica gel 60 (230-400 mesh). Solvents for column chromatography were distilled prior to use. ${ }^{1} \mathrm{H}$ and ${ }^{13} \mathrm{C}$ NMR spectra were recorded with Bruker AMX-400 or Bruker AV-400 spectrometers $(400 \mathrm{MHz}$ for ${ }^{1} \mathrm{H}, 101 \mathrm{MHz}$ for ${ }^{13} \mathrm{C}$ ) and calibrated using the solvent residual peak. In $\mathrm{CDCl}_{3}$ TMS was used for calibration. Melting points were measured with an Büchi melting point M-565. Optical rotations were obtained using a Krüss Optronic P8000 polarimeter $(589 \mathrm{~nm})$. HRMS (ESI) were recorded with a Thermo Finnigan MAT 95XL mass spectrometer.

\section{General procedures}

General procedure 1. Glycosyl chlorides. A solution of the peracetylated disaccharide $(1.0 \mathrm{mmol})$ in acetyl chloride $(7.0$ $\mathrm{mL}$ ) was kept at $0{ }^{\circ} \mathrm{C}$ under argon atmosphere. Dry methanol $(1.0 \mathrm{~mL})$ was added dropwise over a period of 3 hours. After final addition, the solution was allowed to warm slowly to room temperature and stirred until TLC (heptane/acetone $3: 7$ ) indicated no further reaction. The solvent was removed under reduced pressure and the crude product was dissolved in DCM, concentrated again and washed with of diisopropyl ether $(25 \mathrm{~mL})$. The product was used directly according to general procedure 2 without further workup.

General procedure 2. Phase transfer glycosylation. The crude glycosyl chloride (1.0 mmol), TBAHS (1.0 equiv.) and the respective acceptor (1.0-1.1 equiv.) were mixed in DCM (10$15 \mathrm{~mL})$ and an aqueous solution of $\mathrm{K}_{2} \mathrm{CO}_{3}(12.5 \mathrm{~mL}, 1 \mathrm{M})$ was added. The reaction mixture was stirred at room temperature until TLC indicated complete consumption of the donor. The organic phase was separated, dried over $\mathrm{Na}_{2} \mathrm{SO}_{4}$, and the solvent was removed under reduced pressure. The crude product was subjected to column chromatography in the solvent stated.

General procedure 3. Zemplén deacetylation. The starting material $(1.0 \mathrm{mmol})$ was dissolved in $\mathrm{MeOH}(15-20 \mathrm{~mL})$ and was treated with a catalytic amount of sodium methoxide. The solution was stirred until TLC indicated complete consumption of the starting material. If the product has precipitated during this time it was filtered off, otherwise the solution was neutralised with Amberlite IR-120 $\left(\mathrm{H}^{+}\right)$resin and concentrated. The product was dried at $40^{\circ} \mathrm{C}$ under high vacuum.

General procedure 4. Allyl ester deprotection and subsequent decarboxylation. The starting material $(1.0 \mathrm{mmol})$ dissolved in THF $(15 \mathrm{~mL})$ was treated morpholine (10 equiv.) and $\operatorname{Pd}\left(\mathrm{PPh}_{3}\right) 4$ (0.1 equiv.). The solution was stirred overnight at room temperature. After removal of the solvent silver acetate (3 equiv.), potassium carbonate (6-7 equiv.) and acetic anhydride $(10 \mathrm{~mL})$ were added. The resulting mixture was heated to 90-95 ${ }^{\circ} \mathrm{C}$ for $15-20 \mathrm{~min}$. After cooling to room temperature, the mixture was diluted with water and DCM. The organic phase was washed twice with water and once with a diluted aqueous $\mathrm{NaHCO}_{3}$ solution. After drying $\left(\mathrm{Na}_{2} \mathrm{SO}_{4}\right)$ the solvent was removed under reduced pressure and the crude product was subjected to column chromatography in the solvent stated.

4-Nitrophenyl 2-acetamido-4,6-di-O-acetyl-2-deoxy-3-O-(2,3,4, 6-tetra- $O$-acetyl- $\beta$-D-galactopyranosyl)- $\beta$-D-glucopyranoside (4a). Prepared according to general procedures 1 and 2. (1) $2 \mathrm{a}(3.00 \mathrm{~g}$, $4.43 \mathrm{mmol})$, acetyl chloride $(30 \mathrm{~mL})$, methanol $(4.2 \mathrm{~mL})$. TLC (heptane/acetone $3: 7, R_{\mathrm{F}}: 0.45$; starting material 0.40). (2) DCM $(60 \mathrm{~mL})$, aqueous $\mathrm{K}_{2} \mathrm{CO}_{3}$ solution $(60 \mathrm{~mL}, 1 \mathrm{M})$, TBAHS $(1.50 \mathrm{~g}$, $4.42 \mathrm{mmol}$ ), $p$-nitrophenol $(620 \mathrm{mg}, 4.46 \mathrm{mmol})$. Yield $40 \%$ (1.35 g, $1.78 \mathrm{mmol})$; colorless solid; mp $129^{\circ} \mathrm{C}$; $[\alpha]_{\mathrm{D}}^{25}-27.8(c 0.50$ in $\mathrm{CHCl}_{3}$ ); $R_{\mathrm{F}} 0.69$ (heptane/acetone $\left.3: 7\right) ;{ }^{1} \mathrm{H}-\mathrm{NMR}(400 \mathrm{MHz}$, $\left.\mathrm{CDCl}_{3}\right) \delta 8.21-8.16\left(2 \mathrm{H}, \mathrm{m}, \mathrm{H}_{\text {arom }}\right), 7.10-7.04\left(2 \mathrm{H}, \mathrm{m}, \mathrm{H}_{\text {arom }}\right)$, $5.93(1 \mathrm{H}, \mathrm{d}, J=7.5 \mathrm{~Hz}, \mathrm{NH}), 5.71\left(1 \mathrm{H}, \mathrm{d}, J_{1,2}=7.0 \mathrm{~Hz}, \mathrm{H}-1\right), 5.37-$ $5.35\left(1 \mathrm{H}, \mathrm{m}, \mathrm{H}-4^{\prime}\right), 5.12\left(1 \mathrm{H}, \mathrm{dd}, J_{1^{\prime}, 2^{\prime}}=7.8 \mathrm{~Hz}, J_{2^{\prime}, 3^{\prime}}=10.4 \mathrm{~Hz}, \mathrm{H}-\right.$ $\left.2^{\prime}\right), 5.06(1 \mathrm{H}, \mathrm{dd} \sim \mathrm{vt}, \mathrm{H}-4), 4.99\left(1 \mathrm{H}, \mathrm{dd}, J_{2^{\prime}, 3^{\prime}}=10.4 \mathrm{~Hz}, J_{3^{\prime}, 4^{\prime}}=3.4\right.$ $\left.\mathrm{Hz}, \mathrm{H}-3^{\prime}\right), 4.64\left(1 \mathrm{H}, \mathrm{d}, J_{1^{\prime}, 2^{\prime}}=7.8 \mathrm{~Hz}, \mathrm{H}-1^{\prime}\right), 4.57(1 \mathrm{H}, \mathrm{dd} \sim \mathrm{vt}, \mathrm{H}-3)$, $4.24\left(1 \mathrm{H}, \mathrm{dd}, J_{5,6 \mathrm{a}}=6.1 \mathrm{~Hz}, J_{6 \mathrm{a} / \mathrm{b}}=12.3 \mathrm{~Hz}, \mathrm{H}-6 \mathrm{a}\right), 4.19-4.07(3 \mathrm{H}$, m, H-6b, H-6'a/b), 3.98-3.88 (2H, m, H-5, H-5'), $3.67(1 \mathrm{H}$, ddd $\sim$ vdd, H-2), 2.16, 2.09, 20.8, 2.07, 2.00, 1.98 (each 3H, s, C(O) $\left.\mathrm{CH}_{3}\right), 2.02\left(3 \mathrm{H}, \mathrm{s}, \mathrm{NHC}(\mathrm{O}) \mathrm{CH}_{3}\right) ;{ }^{13} \mathrm{C}-\mathrm{NMR}\left(100 \mathrm{MHz}, \mathrm{CDCl}_{3}\right) \delta$ 170.7, 170.4, 170.1, 170.1, 169.1, $169.1\left(C(\mathrm{O}) \mathrm{CH}_{3}\right), 161.4$ $\left(\mathrm{NHC}(\mathrm{O}) \mathrm{CH}_{3}\right), 143.0\left(\mathrm{C}_{\text {quaternary }}\right), 125.7,116.5\left(\mathrm{CH}_{\text {arom }}\right), 100.7(\mathrm{C}-$ 1'), 96.3 (C-1), 76.0 (C-3), 72.4 (C-5), 70.9, 70.8 (C-3', C-5'), 69.4 (C-2'), 68.5 (C-4), 66.8 (C-4'), 62.5 (C-6), $61.0\left(\mathrm{C}-6^{\prime}\right), 56.1$ (C-2), $23.5\left(\mathrm{NHC}(\mathrm{O}) \mathrm{CH}_{3}\right), 20.8,20.8,20.6,20.6,20.5\left(\mathrm{C}(\mathrm{O}) \mathrm{CH}_{3}\right)$; HRMS (ESI) $\mathrm{m} / z$ for $\mathrm{C}_{32} \mathrm{H}_{40} \mathrm{~N}_{2} \mathrm{O}_{19}$ : $[\mathrm{M}+\mathrm{Na}]^{+}$calcd 779.2123 found 779.2134 .

2-Nitrophenyl 2-acetamido-4,6-di-O-acetyl-2-deoxy-3-O-(2,3,4, 6-tetra- $O$-acetyl- $\beta$-D-galactopyranosyl)- $\beta$-D-glucopyranoside $(4 \mathrm{~b})$. Prepared according to general procedures 1 and 2. (1) $2 \mathrm{a}(3.00 \mathrm{~g}$, $4.43 \mathrm{mmol})$, acetyl chloride $(30 \mathrm{~mL})$, methanol $(4.2 \mathrm{~mL})$. TLC (heptane/acetone $3: 7, R_{\mathrm{F}}: 0.45$; starting material 0.40). (2) DCM $(60 \mathrm{~mL})$, aqueous $\mathrm{K}_{2} \mathrm{CO}_{3}$ solution $(60 \mathrm{~mL}, 1 \mathrm{M})$, TBAHS $(1.50 \mathrm{~g}$, $4.42 \mathrm{mmol}), o$-nitrophenol $(620 \mathrm{mg}, 4.46 \mathrm{mmol})$. Yield $34 \%(1.15$ g, $1.52 \mathrm{mmol})$; colorless solid; mp $182-184{ }^{\circ} \mathrm{C}$; [ $\left.\alpha\right]_{\mathrm{D}}^{25}-49.6$ ( c 0.50 in $\mathrm{CHCl}_{3}$ ); $R_{\mathrm{F}} 0.69$ (heptane/acetone $\left.3: 7\right) ;{ }^{1} \mathrm{H}-\mathrm{NMR}(400 \mathrm{MHz}$, $\left.\mathrm{CDCl}_{3}\right) \delta$ 7.80-7.76 (1H, m, $\left.\mathrm{H}_{\text {arom }}\right), 7.54-7.49\left(1 \mathrm{H}, \mathrm{m}, \mathrm{H}_{\text {arom }}\right)$, 7.35-7.32 (1H, m, $\left.\mathrm{H}_{\text {arom }}\right), 7.21-7.26\left(1 \mathrm{H}, \mathrm{m}, \mathrm{H}_{\text {arom }}\right), 5.99(1 \mathrm{H}, \mathrm{bs}$, $\mathrm{NH}), 5.62\left(1 \mathrm{H}, \mathrm{d}, J_{1,2}=8.2 \mathrm{~Hz}, \mathrm{H}-1\right), 5.37-5.34\left(1 \mathrm{H}, \mathrm{m}, \mathrm{H}-4^{\prime}\right), 5.08$ $\left(1 \mathrm{H}, \mathrm{dd}, J_{1^{\prime}, 2^{\prime}}=7.8 \mathrm{~Hz}, J_{2^{\prime}, 3^{\prime}}=10.4 \mathrm{~Hz}, \mathrm{H}-2^{\prime}\right), 5.03-4.97(1 \mathrm{H}, \mathrm{m}$, $\mathrm{H}-4), 4.98\left(1 \mathrm{H}, \mathrm{dd}, J_{2^{\prime}, 3^{\prime}}=10.4 \mathrm{~Hz}, J_{3^{\prime}, 4^{\prime}}=3.8 \mathrm{~Hz}, \mathrm{H}-3^{\prime}\right), 4.75(1 \mathrm{H}$, $\mathrm{dd} \sim \mathrm{vt}, \mathrm{H}-3), 4.58\left(1 \mathrm{H}, \mathrm{d}, J_{1^{\prime}, 2^{\prime}}=7.8 \mathrm{~Hz}, \mathrm{H}-1^{\prime}\right), 4.25\left(1 \mathrm{H}, \mathrm{dd}, J_{5,6 \mathrm{a}}\right.$ $\left.=5.7 \mathrm{~Hz}, J_{6 \mathrm{a} / \mathrm{b}}=12.3 \mathrm{~Hz}, \mathrm{H}-6 \mathrm{a}\right), 4.19\left(1 \mathrm{H}, \mathrm{dd}, J_{5,6 \mathrm{~b}}=2.5 \mathrm{~Hz}, J_{6 \mathrm{a} / \mathrm{b}}\right.$ $=12.3 \mathrm{~Hz}, \mathrm{H}-6 \mathrm{~b}), 4.14-4.09\left(2 \mathrm{H}, \mathrm{m}, \mathrm{H}-6^{\prime} \mathrm{a} / \mathrm{b}\right), 3.92-3.85(2 \mathrm{H}, \mathrm{m}$, 
$\left.\mathrm{H}-5, \mathrm{H}-5^{\prime}\right), 3.45-3.37$ (1H, m, H-2), 2.15, 2.10, 2.08, 2.07, 2.06, 1.98 (each $\left.3 \mathrm{H}, \mathrm{s}, \mathrm{C}(\mathrm{O}) \mathrm{CH}_{3}\right), 2.05$ (3H, s, NHC(O) $\left.\mathrm{CH}_{3}\right) ;{ }^{13} \mathrm{C}-\mathrm{NMR}$ $\left(100 \mathrm{MHz}, \mathrm{CDCl}_{3}\right): \delta=171.9\left(\mathrm{NHC}(\mathrm{O}) \mathrm{CH}_{3}\right), 170.5,170.4,170.2$, 170.1, 169.3, $169.1\left(C(\mathrm{O}) \mathrm{CH}_{3}\right), 149.7,141.1$ ( $\left.\mathrm{C}_{\text {quaternary }}\right), 134.0$, 125.1, 123.4, 119.4 ( $\left.\mathrm{CH}_{\text {arom }}\right), 100.8$ (C-1'), 98.4 (C-1), 76.1 (C-3), 72.4 (C-5), $71.0\left(\mathrm{C}-3^{\prime}\right), 70.7\left(\mathrm{C}-5^{\prime}\right), 69.4\left(\mathrm{C}-2^{\prime}\right), 68.9$ (C-4), 66.9 (C$\left.4^{\prime}\right), 62.3$ (C-6), $61.0\left(\mathrm{C}-6^{\prime}\right), 58.2(\mathrm{C}-2), 23.6\left(\mathrm{NHC}(\mathrm{O}) \mathrm{CH}_{3}\right), 20.9$, 20.8, 20.7, 20.6, 20.6, $20.5\left(\mathrm{C}(\mathrm{O}) \mathrm{CH}_{3}\right)$; HRMS (ESI) $\mathrm{m} / \mathrm{z}$ for $\mathrm{C}_{32} \mathrm{H}_{40} \mathrm{~N}_{2} \mathrm{O}_{19}:[\mathrm{M}+\mathrm{Na}]^{+}$calcd 779.2123 found 779.2139 .

4-Nitrophenyl 2-acetamido-3,6-di-O-acetyl-2-deoxy-4-O-(2,3,4, 6-tetra- $\boldsymbol{O}$-acetyl- $\boldsymbol{\beta}$-D-galactopyranosyl)- $\beta$-D-glucopyranoside (4c). Prepared according to general procedures 1 and 2. (1) 2 b (3.00 g, $4.43 \mathrm{mmol})$, acetyl chloride $(30 \mathrm{~mL})$, methanol $(4.2 \mathrm{~mL})$. TLC (heptane/acetone $3: 7, R_{\mathrm{F}}: 0.52$; starting material 0.43). (2) DCM $(60 \mathrm{~mL})$, aqueous $\mathrm{K}_{2} \mathrm{CO}_{3}$ solution $(60 \mathrm{~mL}, 1 \mathrm{M})$, TBAHS $(1.50 \mathrm{~g}$, $4.42 \mathrm{mmol}$ ), $p$-nitrophenol (620 mg, $4.46 \mathrm{mmol}$ ). Yield $45 \%$ (1.59 g, $2.10 \mathrm{mmol}$ ); colorless solid; mp $156-157{ }^{\circ} \mathrm{C}$ (lit., ${ }^{17} 155-$ $\left.156{ }^{\circ} \mathrm{C}\right) ;[\alpha]_{\mathrm{D}}^{25}-36.2\left(c \quad 0.5\right.$ in $\left.\mathrm{CHCl}_{3}\right)\left(\right.$ lit., ${ }^{17}[\alpha]_{\mathrm{D}}-36.7(c) 1.3$ in $\left.\mathrm{CHCl}_{3}\right)$ ); $R_{\mathrm{F}} 0.47$ (heptane/acetone $3: 7$ ); ${ }^{1} \mathrm{H}-\mathrm{NMR}(400 \mathrm{MHz}$, $\left.\mathrm{CDCl}_{3}\right) \delta$ 8.21-8.17 (2H, m, $\left.\mathrm{H}_{\text {arom }}\right), 7.10-7.05$ (2H, $\mathrm{m}, \mathrm{H}_{\text {arom }}$ ), $6.19(1 \mathrm{H}, \mathrm{d}, J=8.8 \mathrm{~Hz}, \mathrm{NH}), 5.40-5.38\left(1 \mathrm{H}, \mathrm{m}, \mathrm{H}-4^{\prime}\right), 5.30(1 \mathrm{H}, \mathrm{d}$, $\left.J_{1,2}=6.0 \mathrm{~Hz}, \mathrm{H}-1\right), 5.21(1 \mathrm{H}, \mathrm{dd} \sim \mathrm{vt}, \mathrm{H}-3), 5.14\left(1 \mathrm{H}, \mathrm{dd}, J_{1^{\prime}, 2^{\prime}}=7.9\right.$ $\left.\mathrm{Hz}, J_{2^{\prime}, 3^{\prime}}=10.4 \mathrm{~Hz}, \mathrm{H}-2^{\prime}\right), 5.02\left(1 \mathrm{H}, \mathrm{dd}, J_{2^{\prime}, 3^{\prime}}=10.4 \mathrm{~Hz}, J_{3^{\prime}, 4^{\prime}}=3.5\right.$ $\left.\mathrm{Hz}, \mathrm{H}-3^{\prime}\right), 4.51\left(1 \mathrm{H}, \mathrm{d}, J_{1^{\prime}, 2^{\prime}}=7.9 \mathrm{~Hz}, \mathrm{H}-1^{\prime}\right), 4.47\left(1 \mathrm{H}, \mathrm{dd}, J_{5,6 \mathrm{a}}=\right.$ $\left.4.1 \mathrm{~Hz}, J_{6 \mathrm{a} / \mathrm{b}}=12.0 \mathrm{~Hz}, \mathrm{H}-6 \mathrm{a}\right), 4.43-4.37$ (1H, m, H-2), 4.20-4.08 (3H, m, H-6b, H-6'a/b), 3.97-3.90 (2H, m, H-5, H-5'), 3.89-3.85 (1H, m, H-4), 2.14, 2.13, 2.10, 2.06, 2.01, 1.99 (each 3H, s, C(O) $\left.\mathrm{CH}_{3}\right), 2.02\left(\mathrm{NC}(\mathrm{O}) \mathrm{CH}_{3}\right) ;{ }^{13} \mathrm{C}-\mathrm{NMR}\left(100 \mathrm{MHz}, \mathrm{CDCl}_{3}\right) \delta 170.3$, 170.3, 170.2, 170.1, 170.0, 169.9, $169.8\left(\mathrm{C}(\mathrm{O}) \mathrm{CH}_{3}\right), 161.3,142.8$ ( $\left.\mathrm{C}_{\text {quaternary }}\right), 125.7,116.4\left(\mathrm{CH}_{\text {arom }}\right), 100.7$ (C-1'), $97.8(\mathrm{C}-1), 74.0$ (C-4), 72.9 (C-5), $71.0\left(\mathrm{C}-5^{\prime}\right), 70.4$ (C-3, C-3'), 69.0 (C-2'), 66.6 (C$\left.4^{\prime}\right), 62.5$ (C-6), $60.8\left(\mathrm{C}-6^{\prime}\right), 51.1$ (C-2), $23.1\left(\mathrm{NC}(\mathrm{O}) \mathrm{CH}_{3}\right), 20.9$, 20.7, 20.6, 20.6, 20.5, 20.5 (C(O) $\left.\mathrm{CH}_{3}\right)$; HRMS (ESI) $\mathrm{m} / z$ for $\mathrm{C}_{32} \mathrm{H}_{40} \mathrm{~N}_{2} \mathrm{O}_{19}:[\mathrm{M}+\mathrm{Na}]^{+}$calcd 779.2123 found 779.2133 .

2-Nitrophenyl 2-acetamido-3,6-di-O-acetyl-2-deoxy-4-O-(2,3,4, 6-tetra- $\boldsymbol{O}$-acetyl- $\boldsymbol{\beta}$-D-galactopyranosyl)- $\boldsymbol{\beta}$-D-glucopyranoside (4d). Prepared according to general procedures 1 and 2. (1) 2 b (3.00 g, $4.43 \mathrm{mmol})$, acetyl chloride $(30 \mathrm{~mL})$, methanol $(4.2 \mathrm{~mL})$. TLC (heptane/acetone $3: 7, R_{\mathrm{F}}: 0.52$; starting material 0.43). (2) DCM $(60 \mathrm{~mL})$, aqueous $\mathrm{K}_{2} \mathrm{CO}_{3}$ solution $(60 \mathrm{~mL}, 1 \mathrm{M})$, TBAHS $(1.50 \mathrm{~g}$, $4.42 \mathrm{mmol}$ ), $o$-nitrophenol (620 mg, $4.46 \mathrm{mmol}$ ). Yield 44\% (1.49 $\mathrm{g}, 1.97 \mathrm{mmol})$; colorless solid; $\mathrm{mp} 118{ }^{\circ} \mathrm{C}$; $[\alpha]_{\mathrm{D}}^{25}-12.0($ c 0.55 in $\left.\mathrm{CHCl}_{3}\right) ; R_{\mathrm{F}} 0.40$ (heptane/acetone $\left.3: 7\right) ;{ }^{1} \mathrm{H}-\mathrm{NMR}(400 \mathrm{MHz}$, $\left.\mathrm{CDCl}_{3}\right) \delta 7.78\left(1 \mathrm{H}, \mathrm{dd}, \mathrm{H}_{\text {arom }}\right), 7.54-7.48\left(1 \mathrm{H}, \mathrm{m}, \mathrm{H}_{\text {arom }}\right), 7.36-$ $7.32\left(1 \mathrm{H}, \mathrm{m}, \mathrm{H}_{\text {arom }}\right), 7.17-7.12\left(1 \mathrm{H}, \mathrm{m}, \mathrm{H}_{\text {arom }}\right), 6.22(1 \mathrm{H}, \mathrm{d}, J=$ $8.8 \mathrm{~Hz}, \mathrm{NH}$ ), 5.40-5.35 (2H, m, H-1, H-4'), 5.25 (1H, dd $\sim \mathrm{vt}, \mathrm{H}-3$ ), $5.16\left(1 \mathrm{H}, \mathrm{dd}, J_{1^{\prime}, 2^{\prime}}=7.8 \mathrm{~Hz}, J_{2^{\prime}, 3^{\prime}}=10.4 \mathrm{~Hz}, \mathrm{H}-2^{\prime}\right), 5.03(1 \mathrm{H}, \mathrm{dd}$, $\left.J_{2^{\prime}, 3^{\prime}}=10.4 \mathrm{~Hz}, J_{3^{\prime}, 4^{\prime}}=3.5 \mathrm{~Hz}, \mathrm{H}-3^{\prime}\right), 4.54\left(1 \mathrm{H}, \mathrm{dd}, J_{5^{\prime}, 6 \mathrm{a}^{\prime}}=3.8 \mathrm{~Hz}\right.$, $\left.J_{6 \mathrm{a} / \mathrm{b}^{\prime}}=11.6 \mathrm{~Hz}, \mathrm{H}-6 \mathrm{a}\right), 4.52\left(1 \mathrm{H}, J_{1^{\prime}, 2^{\prime}}=7.8 \mathrm{~Hz}, \mathrm{H}-1^{\prime}\right), 4.32-4.22$ (2H, m, H-2, H-6b), 4.20-4.28 (2H, m, H-6'a/b), 3.96-3.88 (3H, m, H-4, H-5, H-5') 2.16, 2.16, 2.08, 2.04, 1.98, 1.98 (each 3H, s, $\left.\mathrm{C}(\mathrm{O}) \mathrm{CH}_{3}\right), 2.05\left(3 \mathrm{H}, \mathrm{s}, \mathrm{NC}(\mathrm{O}) \mathrm{CH}_{3}\right) ;{ }^{13} \mathrm{C}-\mathrm{NMR}\left(100 \mathrm{MHz}, \mathrm{CDCl}_{3}\right) \delta$ 170.5, 170.3, 170.1, 170.0, 169.9, $169.7\left(C(\mathrm{O}) \mathrm{CH}_{3}\right), 149.2,141.2$ ( $\left.\mathrm{C}_{\text {quaternary }}\right), 133.7,125.1,122.9,119.0\left(\mathrm{CH}_{\text {arom }}\right), 100.6\left(\mathrm{C}-1^{\prime}\right)$, 98.4 (C-1), 73.7 (C-4), 72.9 (C-5), $71.0\left(\mathrm{C}-5^{\prime}\right), 70.5$ (C-3'), 69.4 (C-3), $69.0\left(\mathrm{C}-2^{\prime}\right), 66.6\left(\mathrm{C}-4^{\prime}\right), 62.4(\mathrm{C}-6), 60.8\left(\mathrm{C}-6^{\prime}\right), 50.9$ (C-2) 23.1 $\left(\mathrm{NC}(\mathrm{O}) \mathrm{CH}_{3}\right), 20.8,20.7,20.6,20.6,20.6,20.5\left(\mathrm{C}(\mathrm{O}) \mathrm{CH}_{3}\right)$; HRMS
(ESI) $m / z$ for $\mathrm{C}_{32} \mathrm{H}_{40} \mathrm{~N}_{2} \mathrm{O}_{19}:[\mathrm{M}+\mathrm{Na}]^{+}$calcd 779.2123 found 779.2122 .

4-Nitrophenyl 2-acetamido-2-deoxy-3- $O$-( $\beta$-D-galactopyranosyl)- $\beta$-D-glucopyranoside (5a). Prepared according to general procedure 3. 4a (1.20 g, $1.56 \mathrm{mmol})$, $\mathrm{MeOH}(25 \mathrm{~mL})$, cat. NaOMe. Yield 88\% (706 mg, $1.40 \mathrm{mmol}$ ); colorless solid; mp $160{ }^{\circ} \mathrm{C}$ dec (lit., $\left.{ }^{18} 184-186\right) ;[\alpha]_{\mathrm{D}}^{25}-13.4\left(c 0.35\right.$ in $\left.\mathrm{H}_{2} \mathrm{O}\right)\left(\right.$ lit. ${ }^{18}[\alpha]_{\mathrm{D}}^{24}$ -14 (c 0.5 in $\left.\mathrm{H}_{2} \mathrm{O}\right)$ ); ${ }^{1} \mathrm{H}-\mathrm{NMR}\left(400 \mathrm{MHz}, \mathrm{D}_{2} \mathrm{O}\right) \delta 8.29-8.24(2 \mathrm{H}, \mathrm{m}$, $\left.\mathrm{H}_{\text {arom }}\right), 7.25-7.20\left(2 \mathrm{H}, \mathrm{m}, \mathrm{H}_{\text {arom }}\right), 5.39\left(1 \mathrm{H}, \mathrm{d}, J_{1,2}=8.3 \mathrm{~Hz}, \mathrm{H}-1\right)$, $4.52\left(1 \mathrm{H}, \mathrm{d}, J_{1^{\prime}, 2^{\prime}}=7.6 \mathrm{~Hz}, \mathrm{H}-1^{\prime}\right), 4.22\left(1 \mathrm{H}, \mathrm{dd}, J_{1,2}=8.3 \mathrm{~Hz}, J_{2,3}=\right.$ $10.4 \mathrm{~Hz}, \mathrm{H}-2)$, 4.03-3.95 (3H, m, H-3, H-4', $\mathrm{H}-6 \mathrm{a}), 3.89-3.69$ (6H, m, H-4, H-5, H-5', H-6b, H-6'a/b), $3.69\left(1 \mathrm{H}, \mathrm{dd}, J_{2^{\prime}, 3^{\prime}}=10.1 \mathrm{~Hz}\right.$, $\left.J_{3^{\prime}, 4^{\prime}}=3.5 \mathrm{~Hz}, \mathrm{H}-3^{\prime}\right), 3.59\left(1 \mathrm{H}, \mathrm{dd}, J_{1^{\prime}, 2^{\prime}}=7.6 \mathrm{~Hz}, J_{2^{\prime}, 3^{\prime}}=10.1 \mathrm{~Hz}\right.$,

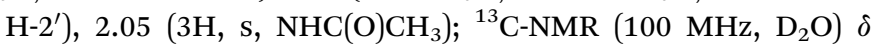
$174.5\left(\mathrm{NHC}(\mathrm{O}) \mathrm{CH}_{3}\right), 161.7,142.7$ ( $\left.\mathrm{C}_{\text {quaternary }}\right), 126.1,116.5$ $\left(\mathrm{CH}_{\text {arom }}\right), 103.5\left(\mathrm{C}-1^{\prime}\right), 98.4(\mathrm{C}-1), 81.8(\mathrm{C}-3), 75.8,75.3\left(\mathrm{C}-5, \mathrm{C}-5^{\prime}\right)$, $72.5\left(\mathrm{C}-3^{\prime}\right), 70.7\left(\mathrm{C}-2^{\prime}\right), 68.6,68.4\left(\mathrm{C}-4, \mathrm{C}-4^{\prime}\right), 61.0\left(\mathrm{C}-6^{\prime}\right), 60.5(\mathrm{C}-$ 6), 54.3 (C-2), 22.1 ( $\left.\mathrm{NHC}(\mathrm{O}) \mathrm{CH}_{3}\right) ;$ HRMS (ESI) $\mathrm{m} / \mathrm{z}$ for $\mathrm{C}_{20} \mathrm{H}_{26} \mathrm{~N}_{2} \mathrm{O}_{13}$ : [M $\left.+\mathrm{Na}\right]^{+}$calcd 527.1489 found 527.1488.

2-Nitrophenyl 2-acetamido-2-deoxy-3-O-( $\beta$-D-galactopyranosyl)- $\boldsymbol{\beta}$-D-glucopyranoside (5b). Prepared according to general procedure 3. 4b (750 mg, $991 \mu \mathrm{mol})$, $\mathrm{MeOH}(15 \mathrm{~mL})$, cat. NaOMe. Yield 80\% (400 mg, $793 \mu \mathrm{mol}$ ); colorless solid; mp 203$204{ }^{\circ} \mathrm{C}$; $[\alpha]_{\mathrm{D}}^{25}-35.7$ (c 0.41 in $\mathrm{H}_{2} \mathrm{O}$ ); ${ }^{1} \mathrm{H}-\mathrm{NMR}\left(400 \mathrm{MHz}, \mathrm{D}_{2} \mathrm{O}\right) \delta$ 7.90-7. 86 (1H, m, $\mathrm{H}_{\text {arom }}$ ), 7.70-7.65 (1H, m, $\left.\mathrm{H}_{\text {arom }}\right), 7.49-7.45$ $\left(1 \mathrm{H}, \mathrm{m}, \mathrm{H}_{\text {arom }}\right), 7.31-7.26\left(1 \mathrm{H}, \mathrm{m}, \mathrm{H}_{\text {arom }}\right), 5.25\left(1 \mathrm{H}, \mathrm{d}, J_{1,2}=8.5\right.$ $\mathrm{Hz}, \mathrm{H}-1), 4.46\left(1 \mathrm{H}, \mathrm{d}, J_{1^{\prime}, 2^{\prime}}=7.6 \mathrm{~Hz}, \mathrm{H}-1^{\prime}\right), 4.18$ (1H, dd $\left.\sim \mathrm{vt}, \mathrm{H}-2\right)$, 4.02-3.97 (1H, m, H-6a), 3.94-3.88 (2H, m, H-3, H-4'), 3.85 (1H, $\left.\mathrm{dd}, J_{5,6 \mathrm{~b}}=4.7 \mathrm{~Hz}, J_{6 \mathrm{a} / \mathrm{b}}=12.3 \mathrm{~Hz}, \mathrm{H}-6 \mathrm{~b}\right), 3.82-3.62(5 \mathrm{H}, \mathrm{m}, \mathrm{H}-4$, $\left.\mathrm{H}-5, \mathrm{H}-5^{\prime}, \mathrm{H}-6^{\prime} \mathrm{a} / \mathrm{b}\right), 3.65\left(1 \mathrm{H}, \mathrm{dd}, J_{2^{\prime}, 3^{\prime}}=10.4 \mathrm{~Hz}, J_{3^{\prime}, 4^{\prime}}=3.8 \mathrm{~Hz}\right.$, $\left.\mathrm{H}-3^{\prime}\right), 3.55$ (1H, dd $\left.\sim \mathrm{vt}, \mathrm{H}-2^{\prime}\right), 2.03\left(3 \mathrm{H}, \mathrm{s}, \mathrm{NHC}(\mathrm{O}) \mathrm{CH}_{3}\right) ;{ }^{13} \mathrm{C}-\mathrm{NMR}$ $\left(100 \mathrm{MHz}, \mathrm{D}_{2} \mathrm{O}\right) \delta 174.8\left(\mathrm{NHC}(\mathrm{O}) \mathrm{CH}_{3}\right), 149.3$ ( $\left.\mathrm{C}_{\text {quaternary }}\right), 134.9$, 125.2, 123.6, $118.2\left(\mathrm{CH}_{\text {arom }}\right), 103.5$ (C-1'), 100.2 (C-1), 81.8 (C-3), 75.9, $75.3\left(\mathrm{C}-5, \mathrm{C}-5^{\prime}\right), 72.5\left(\mathrm{C}-3^{\prime}\right), 70.7\left(\mathrm{C}-2^{\prime}\right), 68.5,68.4\left(\mathrm{C}-4, \mathrm{C}-4^{\prime}\right)$, 61.0 (C-6'), 60.5 (C-6), 54.1 (C-2), 22.1 ( $\left.\mathrm{NHC}(\mathrm{O}) \mathrm{CH}_{3}\right)$; HRMS (ESI) $m / z$ for $\mathrm{C}_{20} \mathrm{H}_{26} \mathrm{~N}_{2} \mathrm{O}_{13}:[\mathrm{M}+\mathrm{Na}]^{+}$calcd 527.1489 found 527.1490 .

4-Nitrophenyl 2-acetamido-2-deoxy-4-O-( $\beta$-D-galactopyranosyl)$\boldsymbol{\beta}$-D-glucopyranoside (5c). Prepared according to general procedure 3. 4c (1.40 g, $1.85 \mathrm{mmol}), \mathrm{MeOH}$ (35 mL), cat. NaOMe. Yield $80 \%$ (750 mg, $1.49 \mathrm{mmol}$ ); colorless solid; mp $213{ }^{\circ} \mathrm{C}$ (lit., ${ }^{19}$ $\left.21{ }^{\circ} \mathrm{C}\right) ;[\alpha]_{\mathrm{D}}^{25}-22.2\left(c 0.5\right.$ in $\left.\mathrm{H}_{2} \mathrm{O}\right)\left(\right.$ lit. ${ }^{19}[\alpha]_{\mathrm{D}}^{25}-18.4\left(c 1\right.$ in $\left.\left.\mathrm{H}_{2} \mathrm{O}\right)\right) ;{ }^{1} \mathrm{H}-$ NMR (400 MHz, $\left.\mathrm{D}_{2} \mathrm{O}\right) \delta 8.28-8.20\left(2 \mathrm{H}, \mathrm{m}, \mathrm{H}_{\text {arom }}\right), 7.23-7.18(2 \mathrm{H}$, $\left.\mathrm{m}, \mathrm{H}_{\text {arom }}\right), 5.37\left(1 \mathrm{H}, \mathrm{d}, J_{1,2}=8.6 \mathrm{~Hz}, \mathrm{H}-1\right), 4.55\left(1 \mathrm{H}, \mathrm{d}, J_{1^{\prime}, 2^{\prime}}=7.8\right.$ $\mathrm{Hz}, \mathrm{H}-1^{\prime}$ ), 4.16-4.10 (1H, m, H-2), 4.10-4.04 (1H, m, H-6a), 3.983.96 (1H, m, H-4'), 3.96-3.86 (4H, m, H-3, H-4, H-5, H-6b), 3.85$3.76\left(3 \mathrm{H}, \mathrm{m}, \mathrm{H}-5^{\prime}, \mathrm{H}-6^{\prime} \mathrm{a} / \mathrm{b}\right), 3.72\left(1 \mathrm{H}, \mathrm{dd}, J_{2^{\prime}, 3^{\prime}}=9.8 \mathrm{~Hz}, J_{3^{\prime}, 4^{\prime}}=3.5\right.$ $\left.\mathrm{Hz}, \mathrm{H}-3^{\prime}\right), 3.61\left(1 \mathrm{H}, \mathrm{dd}, J_{1^{\prime}, 2^{\prime}}=7.8 \mathrm{~Hz}, J_{2^{\prime}, 3^{\prime}}=9.8 \mathrm{~Hz}, \mathrm{H}-2^{\prime}\right), 2.06$ $\left(3 \mathrm{H}, \mathrm{s}, \mathrm{NHC}(\mathrm{O}) \mathrm{CH}_{3}\right) ;{ }^{13} \mathrm{C}-\mathrm{NMR}\left(100 \mathrm{MHz}, \mathrm{D}_{2} \mathrm{O}\right) \delta 174.9\left(\mathrm{C}(\mathrm{O}) \mathrm{CH}_{3}\right)$, 161.7, 142.6 ( $\left.\mathrm{C}_{\text {quaternary }}\right), 126.8,116.5\left(\mathrm{CH}_{\text {arom }}\right), 102.9\left(\mathrm{C}-1^{\prime}\right), 98.5$ (C-1), 78.1 (C-4), 75.4, 75.1 (C-5, C-5'), 72.5 (C-3'), 72.0 (C-3), 71.0 $\left(\mathrm{C}-2^{\prime}\right), 68.6$ (C-4'), $61.6\left(\mathrm{C}-6^{\prime}\right), 59.8$ (C-6), 54.9 (C-2), 22.1 (NHC(O) $\mathrm{CH}_{3}$ ); HRMS (ESI) $m / z$ for $\mathrm{C}_{20} \mathrm{H}_{26} \mathrm{~N}_{2} \mathrm{O}_{13}:[\mathrm{M}+\mathrm{Na}]^{+}$calcd 527.1489 found 527.1496.

2-Nitrophenyl 2-acetamido-2-deoxy-4-O-( $\beta$-D-galactopyranosyl)$\boldsymbol{\beta}$-D-glucopyranoside (5d). Prepared according to general procedure 3 . 4d (1.30 g, $1.72 \mathrm{mmol}), \mathrm{MeOH}$ (25 mL), cat. NaOMe, 
$45{ }^{\circ} \mathrm{C}$. Yield 80\% (698 mg, $\left.1.38 \mathrm{mmol}\right)$; colorless solid; mp 209$212{ }^{\circ} \mathrm{C} ;[\alpha]_{\mathrm{D}}^{22}-27.0\left(c 0.35, \mathrm{H}_{2} \mathrm{O}\right) ;{ }^{1} \mathrm{H}-\mathrm{NMR}\left(400 \mathrm{MHz}, \mathrm{D}_{2} \mathrm{O}\right) \delta 7.89-$ $7.86\left(1 \mathrm{H}, \mathrm{m}, \mathrm{H}_{\text {arom }}\right), 7.69-7.64\left(1 \mathrm{H}, \mathrm{m}, \mathrm{H}_{\text {arom }}\right), 7.47-7.44(1 \mathrm{H}, \mathrm{m}$, $\mathrm{H}_{\text {arom }}$ ), 7.30-7.25 (1H, m, $\left.\mathrm{H}_{\text {arom }}\right), 5.23\left(1 \mathrm{H}, \mathrm{d}, J_{1,2}=8.5 \mathrm{~Hz}, \mathrm{H}-1\right)$, $4.51\left(1 \mathrm{H}, \mathrm{d}, J_{1^{\prime}, 2^{\prime}}=7.9 \mathrm{~Hz}, \mathrm{H}-1^{\prime}\right), 4.08\left(1 \mathrm{H}, \mathrm{dd}, J_{1,2}=8.5 \mathrm{~Hz}, J_{2,3}=\right.$ $10.0 \mathrm{~Hz}, \mathrm{H}-2$ ), 4.06-4.02 (1H, m, H-6a), 3.95-3.89 (2H, m, H-4', H-6b), 3.88-3.73 (6H, m, H-3, H-4, H-5, H-5' , H-6'a/b), 3.68 (1H, $\left.\mathrm{dd}, J_{2^{\prime}, 3^{\prime}}=10.0 \mathrm{~Hz}, J_{3^{\prime}, 4^{\prime}}=3.5 \mathrm{~Hz}, \mathrm{H}-3^{\prime}\right), 3.56\left(1 \mathrm{H}, \mathrm{dd}, J_{1^{\prime}, 2^{\prime}}=7.9\right.$ $\left.\mathrm{Hz}, J_{2^{\prime}, 3^{\prime}}=10.0 \mathrm{~Hz}, \mathrm{H}-2^{\prime}\right), 2.04\left(\mathrm{~s}, 3 \mathrm{H}, \mathrm{NH}\left(\mathrm{C}(\mathrm{O}) \mathrm{CH}_{3}\right)\right) ;{ }^{13} \mathrm{C}-\mathrm{NMR}$ $\left(100 \mathrm{MHz}, \mathrm{D}_{2} \mathrm{O}\right) \delta 174.7\left(C(\mathrm{O}) \mathrm{CH}_{3}\right), 149.3,140.3$ ( $\left.\mathrm{C}_{\text {quaternary }}\right)$, 134.9, 125.1, 123.5, $118.2\left(\mathrm{CH}_{\text {arom }}\right), 102.9\left(\mathrm{C}-1^{\prime}\right), 100.2(\mathrm{C}-1), 77.9$ (C-4), 75.4, $75.2\left(\mathrm{C}-5, \mathrm{C}^{\prime} 5^{\prime}\right), 72.5\left(\mathrm{C}-3^{\prime}\right), 72.1(\mathrm{C}-3), 70.9\left(\mathrm{C}-2^{\prime}\right), 68.5$ $\left(\mathrm{C}-4^{\prime}\right), 61.0\left(\mathrm{C}^{\prime} 6^{\prime}\right), 59.8(\mathrm{C}-6), 54.6(\mathrm{C}-2), 22.1\left(\mathrm{NH}\left(\mathrm{C}(\mathrm{O}) \mathrm{CH}_{3}\right)\right)$; HRMS (ESI) $m / z$ for $\mathrm{C}_{20} \mathrm{H}_{26} \mathrm{~N}_{2} \mathrm{O}_{13}:[\mathrm{M}+\mathrm{Na}]^{+}$calcd 527.1489 found 527.1491 .

(5-Bromo-4-chloro-indox-3-ylic acid allyl ester) 2-acetamido-4,6-di- $O$-acetyl-2-deoxy-3- $O$-(2,3,4,6-tetra- $O$-acetyl- $\beta$-Dgalactopyranosyl)- $\boldsymbol{\beta}$-D-glucopyranoside (6a). Prepared according to general procedures 1 and 2 . (1) $2 \mathrm{a}(1.00 \mathrm{~g}, 1.47 \mathrm{mmol})$, acetyl chloride $(15 \mathrm{~mL})$, methanol $(1.5 \mathrm{~mL})$. TLC (heptane/acetone $3: 7$, $R_{\mathrm{F}}$ : 0.52; starting material 0.43). (2) DCM (17 mL), aqueous $\mathrm{K}_{2} \mathrm{CO}_{3}$ solution (17 mL, $1 \mathrm{M}$ ), TBAHS (520 mg, $1.53 \mathrm{mmol}$ ), 5-bromo-4chloro-indoxylic acid allyl ester (500 mg, $1.51 \mathrm{mmol}$ ). Yield $46 \%$ (635 mg, $0.670 \mathrm{mmol}$ ); colorless solid; mp 152-155 ${ }^{\circ} \mathrm{C}$; $[\alpha]_{\mathrm{D}}^{23}-24.2$ (c $\left.0.5, \mathrm{CHCl}_{3}\right) ; R_{\mathrm{F}} 0.39$ (hexane/acetone $\left.1: 1\right) ;{ }^{1} \mathrm{H}-\mathrm{NMR}(400 \mathrm{MHz}$, $\left.\mathrm{CDCl}_{3}\right) \delta 8.69(1 \mathrm{H}, \mathrm{s}, \mathrm{NH}), 7.49\left(1 \mathrm{H}, \mathrm{d}, J=8.9 \mathrm{~Hz}, \mathrm{H}_{\text {arom }}\right), 7.10(1 \mathrm{H}$, $\left.\mathrm{d}, J=8.9 \mathrm{~Hz}, \mathrm{H}_{\text {arom }}\right), 6.37$ (1H, d, $\left.J=7.6 \mathrm{~Hz}, C 2-\mathrm{NH}\right), 6.09-6.01$ $\left(1 \mathrm{H}, \mathrm{m},-\mathrm{O}-\mathrm{CH}_{2}-\mathrm{CH}=\mathrm{CH}_{2}\right), 5.47-5.43\left(1 \mathrm{H}, \mathrm{m}, \mathrm{O}-\mathrm{CH}_{2}-\mathrm{CH}=\right.$ $\mathrm{CH}_{2} \mathrm{a}$ ), 5.40-5.35 (3H, m, H-1, H-4 $\left.{ }^{\prime}, \mathrm{O}-\mathrm{CH}_{2}-\mathrm{CH}=\mathrm{CH}_{2} \mathrm{~b}\right), 5.09(1 \mathrm{H}$, $\left.\mathrm{dd}, J_{1^{\prime}, 2^{\prime}}=7.9 \mathrm{~Hz}, J_{2^{\prime}, 3^{\prime}}=10.4 \mathrm{~Hz}, \mathrm{H}-2^{\prime}\right), 5.03-4.98\left(2 \mathrm{H}, \mathrm{m}, \mathrm{H}-3^{\prime}, \mathrm{H}-\right.$ 4), $4.70\left(1 \mathrm{H}, \mathrm{d}, J_{1^{\prime}, 2^{\prime}}=7.9 \mathrm{~Hz}, \mathrm{H}-1^{\prime}\right), 4.90-4.83\left(2 \mathrm{H}, \mathrm{m}, \mathrm{O}-\mathrm{CH}_{2}-\right.$ $\left.\mathrm{CH}=\mathrm{CH}_{2}\right), 4.35(1 \mathrm{H}, \mathrm{dd} \sim \mathrm{vt}, \mathrm{H}-3), 4.10\left(1 \mathrm{H}, \mathrm{dd}, J_{5,6 \mathrm{a}}=4.9 \mathrm{~Hz}\right.$, $\left.J_{6 \mathrm{a}, 6 \mathrm{~b}}=12.1 \mathrm{~Hz}, \mathrm{H}-6 \mathrm{a}\right), 4.00\left(1 \mathrm{H}, \mathrm{dd}, J_{5,6 \mathrm{~b}}=3.0 \mathrm{~Hz}, J_{6 \mathrm{a}, 6 \mathrm{~b}}=12.1\right.$ Hz, H-6b), 3.99-3.94 (1H, m, H-2), 3.92-3.88 (1H, m, H-5'), 3.62 $\left(1 \mathrm{H}\right.$, ddd, $\left.J_{4,5}=9.4 \mathrm{~Hz}, J_{5,6 \mathrm{a}}=4.9 \mathrm{~Hz}, J_{5,6 \mathrm{~b}}=3.0 \mathrm{~Hz}, \mathrm{H}-5\right), 2.15$, 2.13, 2.06, 2.03, 1.97, 1.95 (each 3H, s, C(O)CH $\left.\mathrm{CH}_{3}\right), 1.99(3 \mathrm{H}, \mathrm{s}$, $\left.\mathrm{NHC}(\mathrm{O}) \mathrm{CH}_{3}\right) ;{ }^{13} \mathrm{C}-\mathrm{NMR}\left(100 \mathrm{MHz}, \mathrm{CDCl}_{3}\right) \delta 171.2,170.8,170.6$, 170.4, 169.6, $169.4\left(C(\mathrm{O}) \mathrm{CH}_{3}\right), 159.9\left(C(\mathrm{O}) \mathrm{O}-\mathrm{CH}_{2}-\right)$, 140.3, 133.5 $\left(\mathrm{C}_{\text {quaternary }}\right), 131.7\left(\mathrm{O}-\mathrm{CH}_{2}-\mathrm{CH}=\mathrm{CH}_{2}\right), 131.7\left(\mathrm{CH}_{\text {arom }}\right), 127.3,120.9$ ( $\left.\mathrm{C}_{\text {quaternary }}\right), 119.9\left(\mathrm{O}-\mathrm{CH}_{2}-\mathrm{CH}=\mathrm{CH}_{2}\right), 116.9,115.7$ ( $\left.\mathrm{C}_{\text {quaternary }}\right)$, 111.7 ( $\left.\mathrm{CH}_{\text {arom }}\right), 102.1$ (C-1), $100.9\left(\mathrm{C}-1^{\prime}\right), 77.6$ (C-3), 72.3 (C-5), 71.4, 69.4 (C-3', C-4), $70.6\left(\mathrm{C}-5^{\prime}\right), 69.3\left(\mathrm{C}-2^{\prime}\right), 67.1\left(\mathrm{C}^{\prime} 4^{\prime}\right), 66.1\left(\mathrm{O}^{-} \mathrm{CH}_{2}-\right.$ $\left.\mathrm{CH}=\mathrm{CH}_{2}\right), 62.1(\mathrm{C}-6), 61.1\left(\mathrm{C}-6^{\prime}\right), 57.3(\mathrm{C}-2), 23.7\left(\mathrm{NHC}(\mathrm{O}) \mathrm{CH}_{3}\right)$, 21.1, 20.9, 20.8, 20.8, 20.8, $20.7\left(\mathrm{C}(\mathrm{O}) \mathrm{CH}_{3}\right)$; HRMS (ESI) $\mathrm{m} / \mathrm{z}$ for $\mathrm{C}_{38} \mathrm{H}_{44} \mathrm{BrClN}_{2} \mathrm{O}_{19}$ : $[\mathrm{M}+\mathrm{Na}]^{+}$calcd 969.1308 found 969.1293 .

(5-Bromo-4-chloro-indox-3-ylic acid allyl ester) 2acetamido-3,6-di-O-acetyl-2-deoxy-4-O-(2,3,4,6-tetra- $O$-acetyl- $\beta$-Dgalactopyranosyl)- $\boldsymbol{\beta}$-D-glucopyranoside (6b). Prepared according to general procedures 1 and 2. (1) $2 \mathbf{b}$ (900 mg, $1.32 \mathrm{mmol})$, acetyl chloride $(10 \mathrm{~mL})$, methanol $(1.5 \mathrm{~mL})$. TLC (heptane/acetone $3: 7$, $R_{\mathrm{F}}$ : 0.52; starting material 0.43). (2) DCM (15 mL), aqueous $\mathrm{K}_{2} \mathrm{CO}_{3}$ solution (15 mL, $1 \mathrm{M}$ ), TBAHS (414 mg, $1.22 \mathrm{mmol}$ ), 5-bromo-4chloro-indoxylic acid allyl ester (400 mg, $1.21 \mathrm{mmol})$. Yield $45 \%$ (562 mg, $0.593 \mathrm{mmol}$ ); colorless solid; mp 149-152 ${ }^{\circ} \mathrm{C}$; $[\alpha]_{\mathrm{D}}^{23}-7.6$ (c 0.5 in $\mathrm{CHCl}_{3}$ ); $R_{\mathrm{F}} 0.41$ (hexane/acetone $1: 1$ ); ${ }^{1} \mathrm{H}-\mathrm{NMR}$ (400 $\left.\mathrm{MHz} \mathrm{CDCl}_{3}\right) \delta 8.64(1 \mathrm{H}, \mathrm{s}, \mathrm{NH}), 7.48\left(1 \mathrm{H}, \mathrm{d}, J=8.8 \mathrm{~Hz}, \mathrm{H}_{\text {arom }}\right)$, $7.09\left(1 \mathrm{H}, \mathrm{d}, J=8.8 \mathrm{~Hz}, \mathrm{H}_{\text {arom }}\right), 6.58(1 \mathrm{H}, \mathrm{d}, J=8.6 \mathrm{~Hz}, \mathrm{C} 2-\mathrm{NH})$,
6.08-6.01 (1H, m, $\left.-\mathrm{O}-\mathrm{CH}_{2}-\mathrm{CH}=\mathrm{CH}_{2}\right), 5.49-5.45\left(1 \mathrm{H}, \mathrm{m}, \mathrm{O}-\mathrm{CH}_{2}-\right.$ $\left.\mathrm{CH}=\mathrm{CH}_{2} \mathrm{a}\right), \quad 5.41-5.38\left(1 \mathrm{H}, \mathrm{m}, \mathrm{O}-\mathrm{CH}_{2}-\mathrm{CH}=\mathrm{CH}_{2} \mathrm{~b}\right), 5.35-$ $5.33\left(1 \mathrm{H}, \mathrm{m}, \mathrm{H}-4^{\prime}\right), 5.15-5.07$ (3H, m, H-1, H-2', H-3), 4.96 (1H, dd, $\left.J_{2^{\prime}, 3^{\prime}}=10.5 \mathrm{~Hz}, J_{3^{\prime}, 4^{\prime}}=10.5 \mathrm{~Hz}, \mathrm{H}-3^{\prime}\right), 4.89-4.81\left(2 \mathrm{H}, \mathrm{m}, \mathrm{O}-\mathrm{CH}_{2}-\right.$ $\left.\mathrm{CH}=\mathrm{CH}_{2}\right), 4.53\left(1 \mathrm{H}, \mathrm{d}, J_{1^{\prime}, 2^{\prime}}=7.6 \mathrm{~Hz}, \mathrm{H}-1^{\prime}\right), 4.46-4.41(1 \mathrm{H}, \mathrm{m}, \mathrm{H}-$ 2), $4.31\left(1 \mathrm{H}, \mathrm{dd}, J_{5,6 \mathrm{a}}=2.4 \mathrm{~Hz}, J_{6 \mathrm{a}, 6 \mathrm{~b}}=11.8 \mathrm{~Hz}, \mathrm{H}-6 \mathrm{a}\right), 4.15-4.09$ ( $\left.2 \mathrm{H}, \mathrm{m}, \mathrm{H}-6^{\prime} \mathrm{a} / \mathrm{b}\right), 3.93$ (1H, dd $\left.\sim \mathrm{vt}, \mathrm{H}-4\right), 3.90-3.86\left(1 \mathrm{H}, \mathrm{m}, \mathrm{H}-5^{\prime}\right)$, 3.46-3.42 (1H, m, H-5), 2.14, 2.12, 2.06, 1.98 (each 3H, s, C(O) $\left.\mathrm{CH}_{3}\right), 1.99$ (3H, s, NHC(O) $\left.\mathrm{CH}_{3}\right), 1.95\left(6 \mathrm{H}, \mathrm{s}, 2 \mathrm{C}(\mathrm{O}) \mathrm{CH}_{3}\right) ;{ }^{13} \mathrm{C}-\mathrm{NMR}$ $\left(100 \mathrm{MHz}, \mathrm{CDCl}_{3}\right) \delta 170.7,170.6,170.3,170.2,170.1,170.0,169.1$ $\left(C(\mathrm{O}) \mathrm{CH}_{3}\right), 159.4\left(C(\mathrm{O}) \mathrm{O}-\mathrm{CH}_{2}-\right), 141.3,133.2$ ( $\left.\mathrm{C}_{\text {quaternary }}\right), 131.4$ $\left(\mathrm{O}-\mathrm{CH}_{2}-\mathrm{CH}=\mathrm{CH}_{2}\right), 131.0\left(\mathrm{CH}_{\text {arom }}\right), 127.4,120.7$ ( $\left.\mathrm{C}_{\text {quaternary }}\right)$, $120.1\left(\mathrm{O}-\mathrm{CH}_{2}-\mathrm{CH}=\mathrm{CH}_{2}\right), \quad 116.1, \quad 115.7$ ( $\left.\mathrm{C}_{\text {quaternary }}\right), \quad 111.5$ $\left(\mathrm{CH}_{\text {arom }}\right), 103.2$ (C-1), 101.2 (C-1'), 74.1 (C-3), 73.3 (C-4), 72.8 (C-5), $70.9\left(\mathrm{C}-3^{\prime}\right), 70.7\left(\mathrm{C}-5^{\prime}\right), 69.2\left(\mathrm{C}-2^{\prime}\right), 66.6\left(\mathrm{C}-4^{\prime}\right), 66.0\left(\mathrm{O}-\mathrm{CH}_{2}-\mathrm{CH}=\right.$ $\mathrm{CH}_{2}$ ), 61.2 (C-6), $60.8\left(\mathrm{C}-6^{\prime}\right), 54.3$ (C-2), $23.3\left(\mathrm{NHC}(\mathrm{O}) \mathrm{CH}_{3}\right), 20.9$, 20.6, 20.6, 20.6, 20.5 (C(O) $\left.\mathrm{CH}_{3}\right)$; HRMS (ESI) $\mathrm{m} / z$ for $\mathrm{C}_{38} \mathrm{H}_{44} \mathrm{BrClN}_{2} \mathrm{O}_{19}$ : $[\mathrm{M}+\mathrm{Na}]^{+}$calcd 969.1308 found 969.1288 .

( $\mathrm{N}$-Acetyl-5-bromo-4-chloro-indox-3-yl) 2-acetamido-4,6-di-Oacetyl-2-deoxy-3-O-(2,3,4,6-tetra- $O$-acetyl- $\beta$-D-galactopyranosyl)$\boldsymbol{\beta}$-D-glucopyranoside (7a). Prepared according to general procedure 4 . 6a (520 mg, $0.548 \mathrm{mmol})$, THF (10 mL), morpholine $(500 \mu \mathrm{L}, 5.74 \mathrm{mmol}), \mathrm{Pd}\left(\mathrm{PPh}_{3}\right)_{4}(60 \mathrm{mg}, 0.052 \mathrm{mmol}), 15$ min, 90-95 ${ }^{\circ} \mathrm{C}$. Yield $85 \%$ (420 mg, $0.463 \mathrm{mmol}$ ), colorless solid; mp 157-159 ${ }^{\circ} \mathrm{C}$; $[\alpha]_{\mathrm{D}}^{23}-44.0\left(c \quad 0.5\right.$ in $\mathrm{CHCl}_{3}$ ); $R_{\mathrm{F}} 0.36$ (hexane/ acetone $1: 1) ;{ }^{1} \mathrm{H}-\mathrm{NMR}\left(400 \mathrm{MHz}, \mathrm{CDCl}_{3}\right) \delta 8.25(1 \mathrm{H}, \mathrm{d}, J=8.9$ $\left.\mathrm{Hz}, \mathrm{H}_{\text {arom }}\right), 7.55\left(1 \mathrm{H}, \mathrm{d}, J=8.9 \mathrm{~Hz}, \mathrm{H}_{\text {arom }}\right), 7.29(1 \mathrm{H}, \mathrm{s},=\mathrm{CH}-\mathrm{N})$, $5.87(1 \mathrm{H}, \mathrm{d}, J=7.8 \mathrm{~Hz}, \mathrm{C} 2-\mathrm{NH}), 5.41\left(1 \mathrm{H}, \mathrm{d}, J_{1,2}=7.9 \mathrm{~Hz}, \mathrm{H}-1\right)$, 5.37-5.35 (1H, m, H-4 $)$, $5.10\left(1 \mathrm{H}, \mathrm{dd}, J_{1^{\prime}, 2^{\prime}}=7.8 \mathrm{~Hz}, J_{2^{\prime}, 2^{\prime}}=10.5\right.$ $\left.\mathrm{Hz}, \mathrm{H}-2^{\prime}\right), 5.03(1 \mathrm{H}, \mathrm{dd} \sim \mathrm{vt}, \mathrm{H}-4), 4.99\left(1 \mathrm{H}, \mathrm{dd}, J_{2^{\prime}, 3^{\prime}}=10.5 \mathrm{~Hz}\right.$, $\left.J_{3^{\prime}, 4^{\prime}}=3.4 \mathrm{~Hz}, \mathrm{H}-3^{\prime}\right)$, 4.66-4.59 (1H, m, H-3), $4.62\left(1 \mathrm{H}, \mathrm{d}, J_{1^{\prime}, 2^{\prime}}=\right.$ $\left.7.8 \mathrm{~Hz}, \mathrm{H}-1^{\prime}\right), 4.37\left(1 \mathrm{H}, \mathrm{dd}, J_{5,6 \mathrm{a}}=2.7 \mathrm{~Hz}, J_{6 \mathrm{a} / \mathrm{b}}=12.4 \mathrm{~Hz}, \mathrm{H}-6 \mathrm{a}\right)$, $4.18\left(1 \mathrm{H}, \mathrm{dd}, J_{5,6 \mathrm{~b}}=5.5 \mathrm{~Hz}, J_{6 \mathrm{a} / \mathrm{b}}=12.4 \mathrm{~Hz}, \mathrm{H}-6 \mathrm{~b}\right), 4.14-4.08(2 \mathrm{H}$, m, H-6'a/b), 3.92-3.84 (2H, m, H-5, H-5'), 3.66-3.57 (1H, m, H-2), 2.60, 2.16, 2.09, 2.09, 2.08, 2.06, 2.05, 1.98 (each 3H, s, C(O) $\left.\mathrm{CH}_{3}\right)$; ${ }^{13} \mathrm{C}-\mathrm{NMR}\left(100 \mathrm{MHz}, \mathrm{CDCl}_{3}\right) \delta 171.1,170.5,170.4,170.2,170.1$, 169.3, 169.1, $168.2\left(C(\mathrm{O}) \mathrm{CH}_{3}\right), 140.0,133.4$ ( $\left.\mathrm{C}_{\text {quaternary }}\right), 130.5$ $\left(\mathrm{CH}_{\text {arom }}\right), 124.7,122.6,118.4\left(\mathrm{C}_{\text {quaternary }}\right), 116.3\left(\mathrm{CH}_{\text {arom }}\right), 112.4$ (=CH-N), $100.8\left(\mathrm{C}-1^{\prime}\right), 99.4(\mathrm{C}-1), 76.2(\mathrm{C}-3), 72.6(\mathrm{C}-5), 71.0(\mathrm{C}-$ $\left.5^{\prime}\right), 70.7\left(\mathrm{C}-3^{\prime}\right), 69.4,69.0$ (C-2', C-4), 66.9 (C-4'), 62.4 (C-6), 61.0 (C-6'), 57.4 (C-2), 23.8, 23.8, 20.9, 20.8, 20.8, 20.7, 20.6, 20.5 $\left(\mathrm{C}(\mathrm{O}) \mathrm{CH}_{3}\right.$ ); HRMS (ESI) $\mathrm{m} / z$ for $\mathrm{C}_{36} \mathrm{H}_{42} \mathrm{BrClN}_{2} \mathrm{O}_{18}$ : $[\mathrm{M}+\mathrm{Na}]^{+}$ calcd 927.1202 found 927.1194 .

( $\mathrm{N}$-Acetyl-5-bromo-4-chloro-indox-3-yl) 2-acetamido-3,6-di-Oacetyl-2-deoxy-4-O-(2,3,4,6-tetra- $O$-acetyl- $\beta$-D-galactopyranosyl)$\boldsymbol{\beta}$-D-glucopyranoside (7b). Prepared according to general procedure $4.6 \mathrm{~b}(390 \mathrm{mg}, 0.411 \mathrm{mmol})$, THF $(8 \mathrm{~mL})$, morpholine (350 $\mu \mathrm{L}, 4.02 \mathrm{mmol}), \mathrm{Pd}\left(\mathrm{PPh}_{3}\right)_{4}(50 \mathrm{mg}, 0.043 \mathrm{mmol}), 15 \mathrm{~min}$, 90-95 ${ }^{\circ} \mathrm{C}$. Yield $62 \%$ (230 mg, $0.254 \mathrm{mmol}$ ); colorless solid; $\mathrm{mp}$ $146-148{ }^{\circ} \mathrm{C} ;[\alpha]_{\mathrm{D}}^{23}-38.0\left(c 0.25\right.$ in $\left.\mathrm{CHCl}_{3}\right) ; R_{\mathrm{F}} 0.32$ (hexane/acetone $1: 1) ;{ }^{1} \mathrm{H}-\mathrm{NMR}\left(400 \mathrm{MHz}, \mathrm{CDCl}_{3}\right) \delta 8.17(1 \mathrm{H}, \mathrm{d}, J=8.9 \mathrm{~Hz}$, $\left.\mathrm{H}_{\text {arom }}\right), 7.54\left(1 \mathrm{H}, \mathrm{d}, J=8.9 \mathrm{~Hz}, \mathrm{H}_{\text {arom }}\right), 7.44(1 \mathrm{H}, \mathrm{s},=\mathrm{CH}-\mathrm{N}), 6.19$ $(1 \mathrm{H}, \mathrm{d}, J=9.1 \mathrm{~Hz}, C 2-\mathrm{NH}), 5.41-5.38\left(1 \mathrm{H}, \mathrm{m}, \mathrm{H}-4^{\prime}\right), 5.22-5.15$ $\left(2 \mathrm{H}, \mathrm{m}, \mathrm{H}-3, \mathrm{H}-2^{\prime}\right), 5.11\left(1 \mathrm{H}, \mathrm{d}, J_{1,2}=5.4 \mathrm{~Hz}, \mathrm{H}-1\right), 5.05$ (1H, dd, $\left.J_{2^{\prime}, 3^{\prime}}=10.4 \mathrm{~Hz}, J_{3^{\prime}, 4^{\prime}}=3.3 \mathrm{~Hz}, \mathrm{H}-3^{\prime}\right), 4.81\left(1 \mathrm{H}, \mathrm{dd}, J_{5,6 \mathrm{a}}=4.7 \mathrm{~Hz}\right.$, $\left.J_{6 \mathrm{a} / \mathrm{b}}=11.8 \mathrm{~Hz}, \mathrm{H}-6 \mathrm{a}\right), 4.57\left(1 \mathrm{H}, \mathrm{d}, J_{1^{\prime}, 2^{\prime}}=8.0 \mathrm{~Hz}, \mathrm{H}-1^{\prime}\right), 4.44-4.39$ $(1 \mathrm{H}, \mathrm{m}, \mathrm{H}-2), 4.24\left(1 \mathrm{H}, \mathrm{dd}, J_{5,6 \mathrm{~b}}=5.0 \mathrm{~Hz}, J_{6 \mathrm{a} / \mathrm{b}}=11.8 \mathrm{~Hz}, \mathrm{H}-6 \mathrm{~b}\right)$, 
$4.19\left(1 \mathrm{H}, \mathrm{dd}, J_{5^{\prime}, 6^{\prime} \mathrm{a}}=6.2 \mathrm{~Hz}, J_{6^{\prime} \mathrm{a} / \mathrm{b}}=11.3 \mathrm{~Hz}, \mathrm{H}-6^{\prime} \mathrm{a}\right), 4.12(1 \mathrm{H}, \mathrm{dd}$, $\left.J_{5^{\prime}, 6^{\prime} \mathrm{b}}=7.1 \mathrm{~Hz}, J_{6^{\prime} \mathrm{a} / \mathrm{b}}=11.3 \mathrm{~Hz}, \mathrm{H}-6^{\prime} \mathrm{b}\right), 3.97-3.87(3 \mathrm{H}, \mathrm{m}, \mathrm{H}-4, \mathrm{H}-$ 5, H-5'), 2.17, 2.09, 1.99 (each 3H, s, C $(\mathrm{O}) \mathrm{CH}_{3}$ ), 2.08, 2.06 (each $\left.6 \mathrm{H}, \mathrm{s}, 2 \mathrm{C}(\mathrm{O}) \mathrm{CH}_{3}\right) ;{ }^{13} \mathrm{C}-\mathrm{NMR}\left(100 \mathrm{MHz}, \mathrm{CDCl}_{3}\right) \delta 170.4,170.4$, 170.3, 170.1, 170.0, 170.0, 169.7, $168.6\left(C(\mathrm{O}) \mathrm{CH}_{3}\right), 139.4,133.5$ ( $\left.\mathrm{C}_{\text {quaternary }}\right), 130.4\left(\mathrm{CH}_{\text {arom }}\right), 118.3$ ( $\left.\mathrm{C}_{\text {quaternary }}\right), 116.3\left(\mathrm{CH}_{\text {arom }}\right)$, 111.8 (=CH-N), $100.8\left(\mathrm{C}-1^{\prime}\right), 99.7(\mathrm{C}-1), 73.8,73.1$ (C-4, C-5), $71.0\left(\mathrm{C}-5^{\prime}\right), 70.5\left(\mathrm{C}-3^{\prime}\right), 70.0(\mathrm{C}-3), 69.1\left(\mathrm{C}-2^{\prime}\right), 66.6\left(\mathrm{C}-4^{\prime}\right), 62.3(\mathrm{C}-$ 6), 60.8 (C-6'), 50.3 (C-2), 23.9, 23.2, 20.8, 20.7, 20.6, 20.5 (C(O) $\mathrm{CH}_{3}$ ); HRMS (ESI) $m / z$ for $\mathrm{C}_{36} \mathrm{H}_{42} \mathrm{BrClN}_{2} \mathrm{O}_{18}:[\mathrm{M}+\mathrm{Na}]^{+}$calcd 927.1202 found 927.1187 .

(5-Bromo-4-chloro-indox-3-yl) 2-acetamido-2-deoxy-3- $O$-( $\beta$-Dgalactopyranosyl)- $\boldsymbol{\beta}$-D-glucopyranoside (8a). Prepared according to general procedure 3. 7a (100 mg, $0.110 \mathrm{mmol}), \mathrm{MeOH}(3 \mathrm{~mL})$, cat. NaOMe, rt. Yield 75\% (51 mg, $0.083 \mathrm{mmol}$ ); colorless solid; mp $230{ }^{\circ} \mathrm{C}$ dec; $[\alpha]_{\mathrm{D}}^{25}-100.8$ (c 0.25 in 50\% DMF/ $\left.\mathrm{H}_{2} \mathrm{O}\right) ;{ }^{1} \mathrm{H}-\mathrm{NMR}$ $\left(400 \mathrm{MHz}, \mathrm{DMSO}^{\left.-\mathrm{d}_{6}\right)} \delta 11.15(1 \mathrm{H}, \mathrm{bs}, \mathrm{NH}), 7.91(1 \mathrm{H}, \mathrm{d}, J=8.3\right.$ $\mathrm{Hz}, \mathrm{C} 2-\mathrm{NH}), 7.36-7.16\left(3 \mathrm{H}, \mathrm{m}, 2 \mathrm{H}_{\mathrm{arom}}=\mathrm{CH}-\mathrm{N}\right), 4.87(1 \mathrm{H}, \mathrm{d}$, $\left.J_{1,2}=8.5 \mathrm{~Hz}, \mathrm{H}-1\right)$, 4.83-4.70 (3H, m, $\left.3 \mathrm{OH}\right), 4.66(1 \mathrm{H}, \mathrm{bs}, \mathrm{OH})$, $4.50(1 \mathrm{H}, \mathrm{bs}, \mathrm{OH}), 4.33(1 \mathrm{H}, \mathrm{bs}, \mathrm{OH}), 4.15\left(1 \mathrm{H}, \mathrm{d}, J_{1^{\prime}, 2^{\prime}}=6.6 \mathrm{~Hz}\right.$, H-1'), 3.85-3.75 (2H, m, H-2, H-6a), 3.66-3.58 (2H, m, H-3, H-4'), 3.57-3.49 (3H, m, H-6b, H-6'a/b), 3.49-3.31 (5H, m, H-2', H-3', $\left.\mathrm{H}-4, \mathrm{H}-5, \mathrm{H}-5^{\prime}\right), 1.82$ (3H, s, NHC(O)CH$)$ ) ${ }^{13} \mathrm{C}-\mathrm{NMR}(100 \mathrm{MHz}$, DMSO-d $\left._{6}\right) \delta 169.9\left(C(\mathrm{O}) \mathrm{CH}_{3}\right), 132.9\left(\mathrm{C}_{\text {quaternary }}\right), 125.5\left(\mathrm{CH}_{\text {arom }}\right)$, 122.9, 117.6 ( $\left.\mathrm{C}_{\text {quaternary }}\right), 114.0,112.3\left(\mathrm{CH}_{\text {arom }},=\mathrm{CH}-\mathrm{N}\right), 111.7$ (C quaternary $), 104.0\left(\mathrm{C}-1^{\prime}\right), 101.7$ (C-1), 84.6 (C-3), 76.8, 75.8 (C-5, C-5'), 72.9, 68.9 (C-3', $\mathrm{C}-4), 70.6\left(\mathrm{C}-2^{\prime}\right), 68.2\left(\mathrm{C}-4^{\prime}\right), 60.8,60.6(\mathrm{C}-6$, C-6'), $54.2(\mathrm{C}-2), 23.1\left(\mathrm{C}(\mathrm{O}) \mathrm{CH}_{3}\right) \mathrm{ppm}$.

(5-Bromo-4-chloro-indox-3-yl) 2-acetamido-2-deoxy-4- $O$-( $\beta$-Dgalactopyranosyl)- $\boldsymbol{\beta}$-D-glucopyranoside (8b). Prepared according to general procedure 3. $7 \mathbf{b}(95 \mathrm{mg}, 0.10 \mathrm{mmol}), \mathrm{MeOH}(3 \mathrm{~mL})$, cat. NaOMe, rt. Yield 73\% (45 mg, $0.073 \mathrm{mmol}$ ); colorless solid; mp $230{ }^{\circ} \mathrm{C}$ dec; $[\alpha]_{\mathrm{D}}^{23}-43.8$ (c 0.5 in 50\% DMF/ $\mathrm{H}_{2} \mathrm{O}$ ); ${ }^{1} \mathrm{H}-\mathrm{NMR}$ $\left(400 \mathrm{MHz}, \mathrm{DMSO}-\mathrm{d}_{6}\right) \delta 11.12(1 \mathrm{H}, \mathrm{bs}, \mathrm{NH}), 7.88(1 \mathrm{H}, \mathrm{d}, J=9.2$ $\mathrm{Hz}, \mathrm{C} 2-\mathrm{NH}), 7.31\left(1 \mathrm{H}, \mathrm{d}, J=8.8 \mathrm{~Hz}, \mathrm{H}_{\text {arom }}\right), 7.29(\mathrm{~s}, 1 \mathrm{H},=\mathrm{CH}-$ $\mathrm{N}), 7.22\left(1 \mathrm{H}, \mathrm{d}, J=8.8 \mathrm{~Hz}, \mathrm{H}_{\text {arom }}\right), 5.07(1 \mathrm{H}, \mathrm{bs}, \mathrm{OH}), 4.79(1 \mathrm{H}$, bs, OH), $4.75(1 \mathrm{H}, \mathrm{t}, \mathrm{OH}), 4.72(1 \mathrm{H}, \mathrm{bs}, \mathrm{OH}), 4.71\left(1 \mathrm{H}, \mathrm{d}, J_{1,2}=\right.$ $8.6 \mathrm{~Hz}, \mathrm{H}-1), 4.67$ (1H, t, OH), $4.51(1 \mathrm{H}, \mathrm{d}, \mathrm{OH}), 4.23\left(1 \mathrm{H}, \mathrm{d}, J_{1^{\prime}, 2^{\prime}}\right.$ $\left.=7.4 \mathrm{~Hz}, \mathrm{H}-1^{\prime}\right), 3.93-3.88(1 \mathrm{H}, \mathrm{m}, \mathrm{H}-6 \mathrm{a}), 3.87-3.81(1 \mathrm{H}, \mathrm{m}, \mathrm{H}-$ 2), 3.68-3.60 (2H, m, H-4', $\mathrm{H}-6 \mathrm{~b}), 3.56$ (1H, dd $\sim \mathrm{vt}, \mathrm{H}-3), 3.54-$ 3.50 (2H, m, H-6 $\mathrm{a} / \mathrm{b}), 3.49-3.42(1 \mathrm{H}, \mathrm{m}, \mathrm{H}-5), 3.40$ (1H, dd $\sim \mathrm{vt}$, $\mathrm{H}-4), 3.34-3.32$ (2H, m, H-2', H-3') $1.82\left(3 \mathrm{H}, \mathrm{s}, \mathrm{NHC}(\mathrm{O}) \mathrm{CH}_{3}\right)$; ${ }^{13} \mathrm{C}-\mathrm{NMR}\left(100 \mathrm{MHz}, \mathrm{DMSO}-\mathrm{d}_{6}\right) \delta 168.7\left(C(\mathrm{O}) \mathrm{CH}_{3}\right), 136.9,132.9$ $\left(\mathrm{C}_{\text {quaternary }}\right), 125.5\left(\mathrm{CH}_{\text {arom }}\right), 123.0,117.6\left(\mathrm{C}_{\text {quaternary }}\right), 114.0$ (= $\mathrm{CH}-\mathrm{N}), 112.3\left(\mathrm{CH}_{\text {arom }}\right), 111.6\left(\mathrm{C}_{\text {quaternary }}\right), 104.0\left(\mathrm{C}-1^{\prime}\right), 102.0$ (C-1), 81.4 (C-4), 75.6, 75.4 (C-5, C-5'), 73.2, $70.6\left(\mathrm{C}-2^{\prime}, \mathrm{C}-3^{\prime}\right)$,
72.3 (C-3), 68.2 (C-4'), 60.5, 60.4 (C-6, C-6'), 54.6 (C-2), 23.1 $\left(\mathrm{C}(\mathrm{O}) \mathrm{CH}_{3}\right)$ ppm.

\section{Acknowledgements}

Support of this work by Glycom A/S, Copenhagen, Denmark, is gratefully acknowledged.

\section{References}

1 R. M. Erney, W. T. Malone, M. B. Skelding, A. A. Marcon, K. M. Kleman-Leyer, M. L. O'Ryan, G. Ruiz-Palacios, M. D. Hilty, L. K. L. K. Pickering and P. A. Prieto, J. Pediatr. Gastroenterol. Nutr., 2000, 30, 181-192.

2 B. La Ferla, D. Prosperi, L. Lay, G. Russo and L. Panza, Carbohydr. Res., 2002, 337, 1333-1342.

3 J. L. Magnani, Arch. Biochem. Biophys., 2004, 426, 122-131.

4 K. Lau, H. Yu, V. Thon, Z. Khedri, M. E. Leon, B. K. Tran and X. Chen, Org. Biomol. Chem., 2011, 9, 2784-2789.

5 S. C. Stocks, M. Albrechtsen and M. A. Kerr, Biochem. J., 1990, 268, 275-280.

6 R. S. Loka, C. M. Sadek, N. A. Romaniuk and C. W. Cairo, Bioconjugate Chem., 2010, 21, 1842-1849.

7 D. Garrido, D. C. Dallas and D. A. Mills, Microbiology, 2013, 159, 649-664.

8 J. A. Kiernan, Biotech. Histochem., 2007, 82, 73-103.

9 J. H. Miller, Experiments in Molecular Genetics, Cold Spring Harbor Laboratory Press, Cold Spring Harbor, NY, 1972.

10 D. Mahrun, Clin. Chim. Acta, 1976, 73, 453-461.

11 S. Tokutake, N. Yamaji and M. Kato, Chem. Pharm. Bull., 1990, 38, 13-18.

12 K. Clinch, G. B. Evans, R. H. Furneaux, P. M. Rendle, P. L. Rhodes, A. M. Roberton, D. I. Rosendale, P. C. Tyler and D. P. Wright, Carbohydr. Res., 2002, 337, 1095-1111.

13 G. Zemplén, Ber. Dtsch. Chem. Ges. B, 1926, 59B, 1254-1266. 14 S. Böttcher, M. Hederos, E. Champion, G. Dekany and J. Thiem, Org. Lett., 2013, 15, 3766-3769.

15 S. Böttcher and J. Thiem, Eur. J. Org. Chem., 2014, 564-574.

16 H. Kunz and H. Waldmann, Angew. Chem., Int. Ed. Engl., 1984, 23, 71-72.

17 S. S. Rana, J. J. Barlow and K. L. Matta, Carbohydr. Res., 1983, 113, 257-271.

18 H. Ohi, S. Kubota and T. Usui, Carbohydr. Res., 1993, 244, 315-323.

19 K. L. Matta and J. J. Barlow, Carbohydr. Res., 1975, 75, 299304. 